\title{
Formation and structure of V-Zr amorphous alloy thin films
}

\author{
D.J.M. King ${ }^{1,2}$, S.C. Middleburgh ${ }^{1}$, A.C.Y. Liu ${ }^{3}$, H.A. Tahini ${ }^{4}$, G.R. Lumpkin ${ }^{1}$, M.B. Cortie ${ }^{2}$ \\ ${ }^{1}$ IME, Australian Nuclear Science and Technology Organisation, \\ Lucas Heights, New South Wales, Australia \\ 2 Institute for Nanoscale Technology, University of Technology, Sydney, New South Wales, Australia \\ ${ }^{3}$ Centre for Electron Microscopy, Monash University, Melbourne, Victoria, Australia \\ ${ }^{4}$ PSE Division, KAUST, Thuwal 23955-6900, Kingdom of Saudi Arabia
}

\begin{abstract}
Although the equilibrium phase diagram predicts that alloys in the central part of the $\mathrm{V}-\mathrm{Zr}$ system should consist of $\mathrm{V}_{2} \mathrm{Zr}$ Laves phase with partial segregation of one element, it is known that under non-equilibrium conditions these materials can form amorphous structures. Here we examine the structures and stabilities of thin film $\mathrm{V}-\mathrm{Zr}$ alloys deposited at room temperature by magnetron sputtering. The films were characterized by X-ray diffraction, transmission electron microscopy and computational methods. Atomic scale modelling was used to investigate the enthalpies of formation of the various competing structures. The calculations confirmed that an amorphous solid solution would be significantly more stable than a random body-centred solid solution of the elements, in agreement with the experimental results. In addition, the modelling effort provided insight into the probable atomic configurations of the amorphous structures allowing predictions of the average distance to first and second nearest neighbours in the system.
\end{abstract}

\section{Keywords}

Amorphous alloy; Density functional theory; Thin films; X-ray diffraction; Zirconium alloys

\section{Introduction}

Tailored V-Zr alloys may potentially be relevant to the energy, chemical, biomedical and nuclear industries. This is because these two individual elements have good creep resistance [1], high melting temperatures, high thermal conductivities, tailorable low densities, relatively small thermal neutron cross-sections [2], and low thermal expansion [3]. Zirconium-based alloys are already extensively used for structural, in-core applications for the current generation of fission reactors [4], while vanadium-based alloys are under consideration for structural components in fusion reactor environments, particularly the first-wall blanket [5]. However, there has been little consideration, so far in the nuclear industry and elsewhere, for binary alloys which simultaneously contain both $\mathrm{V}$ and $\mathrm{Zr}$ in significant proportions.

The binary metallic V-Zr system has been studied both experimentally $[6,7,8]$ and computationally $[9,10,11,12$, 13]. In its elemental form $\mathrm{V}$ is body-centred cubic (BCC) while $\mathrm{Zr}$ is hexagonal-close packed (HCP) below $1136 \mathrm{~K}$ and BCC above that temperature [14]. Although they share the same structure at elevated temperatures, HumeRothery's rules for solid solution formation [15] predict that $\mathrm{V}$ and $\mathrm{Zr}$ should not be mutually soluble because their atomic radii differ by $>15 \%$ and their electro-negativities are dissimilar. Indeed, on normal solidification from the melt they form an intermetallic compound, $\mathrm{V}_{2} \mathrm{Zr}$, by peritectic transformation at $\sim 1573 \mathrm{~K}$, which has the C15 Laves phase structure [16].

Here, we are interested in thin films produced by physical vapour deposition and which contain between 33 and 66 at.\% V. Normally, such films are amorphous or glassy when produced at low temperatures [7], (see Figure 1). It might be expected that amorphous binary films (designated here as $\left.(\mathrm{V}, \mathrm{Zr})_{\mathrm{am}}\right)$ would crystallize to a product containing $\mathrm{V}_{2} \mathrm{Zr}$ plus an element when heated, but instead such samples are reported to crystallise into a dual-phase mixture of elemental $\mathrm{V}$ and $\mathrm{Zr}$ phases, ostensibly because of a difficulty nucleating the complex Laves phase structure at relatively low temperatures [7].

There is, however, another possible explanation for the absence of $\mathrm{V}_{2} \mathrm{Zr}$ in these samples. It may simply be that $\mathrm{V}_{2} \mathrm{Zr}$ is not thermodynamically stable below some limiting temperature and instead, below that temperature, a dualphase mixture of the elements is preferred. Formation of $\sigma$-phase in the Cr-Fe system would be an analogous example, where a mixture of two BCC elemental solid-solutions is more stable below $\sim 773 \mathrm{~K}$ than $\sigma$-phase [17]. There is some theoretical support for this phenomenon occurring in $\mathrm{V}-\mathrm{Zr}$, with recent density functional theory (DFT) calculations of the enthalpy of formation for $\mathrm{V}_{2} \mathrm{Zr}$ to be slightly positive [12] relative to its constituent elements. 
The experimentally observed stability of $\mathrm{V}_{2} \mathrm{Zr}$ at elevated temperatures may be due to the effect of vibrational entropy shifting the free energy of the system in favour of the intermetallic [10].

If thin film coatings of $(\mathrm{V}, \mathrm{Zr})_{\text {am }}$ were to be used in extreme environments then it would be important to know what structural changes they might be susceptible to. In particular, it has been suggested that displacive radiation damage, for example, can accelerate the transformation of metastable metallic solid solutions into their more thermodynamically stable states [18]. Therefore, this study aims to computationally model the various configurations of three stoichiometries: $2 \mathrm{~V}: 1 \mathrm{Zr}, 1 \mathrm{~V}: 1 \mathrm{Zr}$ and $1 \mathrm{~V}: 2 \mathrm{Zr}$ as crystalline and amorphous systems, benchmarking these predictions with experimentally acquired data. The structures are then used to theoretically predict the variation in stability of the amorphous and crystalline systems.

\section{Methodology}

\subsection{Computational Methods}

A plane wave DFT package, VASP [19, 20], was chosen as it will reproduce the intermetallic/metallic nature of the bonding to satisfactory accuracy. It has been successfully used in the past to model intermetallic systems by a number of groups $[21,22,23]$. A $3 \times 3 \times 3 k$-point grid with a Methfessel-Paxton smearing method $(0.125 \mathrm{eV})$ was used. The constant pressure calculations included the GGA-PBE exchange correlation functional, and spinpolarization effects. The semi-core $\mathrm{p}$ states for $\mathrm{V}$, and semi-core $\mathrm{s}$ and $\mathrm{p}$ states, were treated as valence states for Zr. This was achieved by using the supplied plane augmented wave pseudopotentials within the VASP package. The energy convergence criteria for all calculations was within $10^{-4} \mathrm{eV}$ with the exception of the amorphous calculations which was increased to $10^{-3} \mathrm{eV}$.

Calculations were performed for BCC, amorphous and dual-phase structures of the three compositions $2 \mathrm{~V}: 1 \mathrm{Zr}$, $1 \mathrm{~V}: 1 \mathrm{Zr}$ and $1 \mathrm{~V}: 2 \mathrm{Zr}$ as well as for the pure elements. BCC Zr's lattice energy was $0.09 \mathrm{eV} /$ atom higher than HCP Zr, as expected [16]. Formation enthalpies were computed for both crystalline and amorphous structures to understand their relative stabilities.

Further investigations were made on these systems using supercells consisting of 48-54 atoms. The formation enthalpies for the $(\mathrm{V}, \mathrm{Zr})_{\mathrm{am}}$ and complete BCC solid solution, denoted as $(\mathrm{V}, \mathrm{Zr})_{\mathrm{BCC}}$, phases were averaged across 10 unique supercells [23] and compared to $\mathrm{V}_{2} \mathrm{Zr}$ and dualphase $\mathrm{V}_{2} \mathrm{Zr}+\mathrm{Zr}$ formation enthalpies. The random nature of the amorphous systems were simulated by randomly assigning $\mathrm{V}$ and $\mathrm{Zr}$ to $\mathrm{x}, \mathrm{y}, \mathrm{z}$ co-ordinates with a restriction of no atoms placed within $1.5 \AA$ of each other. Supercells of BCC solid solutions were created by randomly assigning $\mathrm{V}$ and $\mathrm{Zr}$ atoms to BCC lattice sites. By averaging the result over multiple random supercells, similar results to the special quasi-random structure (SQS) method will be attained as previously highlighted in a number of studies $[24,25]$.

The geometrically optimised amorphous supercells were then analysed producing X-ray diffraction (XRD) simulations and radial distribution functions (RDF), within the Reflex and Forcite modules, respectively. The parameters mirrored the experimental values, see 2.2.2. The central peak location (CPL) of XRD and RDF data were determined from fitted Voigt profiles and Gaussian functions, respectively. Bragg's Law was used to calculate the equivalent d-space value from the $2 \theta$ values. The volume per atom in each system was calculated as $\frac{\sqrt[3]{V_{a t}}}{2}$ where $V_{a t}$ is the total supercell volume divided by the number of atoms within the supercell after optimisation. This is to give a more meaningful representation of volume as an approximated average radius.

\subsection{Experimental Methods \\ 2.2.1. Thin Film Preparation}

Crystalline silicon wafers were cleaned using sonication for 15 minutes in ethanol and used as substrates on a rotating stage. The vacuum chamber reached a base pressure of $\sim 4 \times 10^{-5} \mathrm{~Pa}$ before an Ar partial pressure of $2.8 \times 10^{-1} \mathrm{~Pa}$ was established. Two magnetron guns mounted with targets of $99.999 \% \mathrm{~V}$ and $99.999 \% \mathrm{Zr}$ were used to co-deposit $\sim 200 \mathrm{~nm}$ thick thin films at room temperature.

\subsubsection{X-ray Diffraction}

X-ray diffraction patterns were measured using a BRUKER D8 instrument, with weighted $\mathrm{Cu} \mathrm{Ka}$ radiation, in an angular rangeof $10-70^{\circ}$ two theta with a step size of $0.03^{\circ}$ at 20 seconds each on a rotating stage.

\subsubsection{SEM-EDS Analysis}

A JEOL JSM6300 scanning electron microscope (SEM, JEOL Ltd., Tokyo, Japan) operating at $15 \mathrm{kV}$ and equipped with a Noran Voyager Series IV energy-dispersive spectroscopy system (EDS, Thermo Electron Corporation) was used for microstructural and phase-composition analysis [26]. Ten random spectra were obtained from each sample and the compositions were then averaged.

\subsubsection{Transmission Electron Microscopy}

Fragments of the thin films were scratched off the $\mathrm{Si}$ substrate using a diamond scribe and ground under ethanol in a mortar and pestle. The dispersion was ultrasonicated and allowed to settle for 5 minutes. Three drops of the top fraction were dropped onto holey carbon grids for observation in the transmission electron microscope (TEM). Selected area diffraction patterns were obtained using a JEOL $2010 \mathrm{~F}$ operated at $200 \mathrm{kV}$. The grains were monitored via bright field images and selected area electron diffraction patterns. The grains were also monitored at elevated temperature using a Gatan, double tilt heating stage, Model 652, to conduct controlled temperature experiments between 300 and $773 \mathrm{~K}$. High resolution brightfield scanning transmission electron microscopy (BF-STEM) 
Table 1: Composition of experimental thin film and computationally modelled amorphous alloys. The inter-atomic distances are calculated by determining the XRD pattern (experimental and simulated) central peak location.

\begin{tabular}{cccc}
\hline System & $\begin{array}{c}\text { V content } \\
\text { (at.\%) }\end{array}$ & $\begin{array}{c}\text { Zr content } \\
\text { (at.\%) }\end{array}$ & $\begin{array}{c}\text { Inter-atomic } \\
\text { distance }(\AA)\end{array}$ \\
\hline $\begin{array}{c}\text { Experiment } \\
\text { 2V:1Zr }\end{array}$ & 72.22 & 27.78 & 2.29 \\
$1 \mathrm{~V}: 1 \mathrm{Zr}$ & 53.05 & 46.95 & 2.40 \\
$1 \mathrm{~V}: 2 \mathrm{Zr}$ & 35.06 & 64.94 & 2.46 \\
& & & \\
Model & & & \\
$2 \mathrm{~V}: 1 \mathrm{Zr}$ & 66.67 & 33.33 & 2.33 \\
$1 \mathrm{~V}: 1 \mathrm{Zr}$ & 50.00 & 50.00 & 2.39 \\
$1 \mathrm{~V}: 2 \mathrm{Zr}$ & 33.33 & 66.67 & 2.44 \\
\hline
\end{tabular}

was performed in a double-corrected FEI Titan ${ }^{3}$ 80-300 FEGTEM operated at $300 \mathrm{keV}$ with a convergence angle of $21 \mathrm{mrad}$ and a camera length of $91 \mathrm{~mm}$.

\section{Results}

\subsection{Thin Film Characterisation}

In this section we describe the results of co-depositing $\mathrm{V}$ and $\mathrm{Zr}$ onto Si wafers. The results from the films are used to validate the simulation methods used subsequently in this work.

SEM-EDS analysis of the three films was used to accurately determine the composition of the specimens and compare them to their target compositions of $2 \mathrm{~V}: 1 \mathrm{Zr}, 1 \mathrm{~V}: 1 \mathrm{Zr}$ and $1 \mathrm{~V}: 2 \mathrm{Zr}$, see Table 1 . The target compositions were roughly achieved, the largest deviation was found to be for the $2 \mathrm{~V}: 1 \mathrm{Zr}$ composition, which was found to have a $\mathrm{V}$ to $\mathrm{Zr}$ ratio of $2.6: 1$ instead of $2: 1$. This variation, although significant, was accounted for in the subsequent results and analysis.

Grazing-incidence XRD was used to understand the thin films' crystal structure. As expected from the literature [7], the equilibrium crystalline phases: BCC structured $\mathrm{V}$, Laves phase $\mathrm{V}_{2} \mathrm{Zr}$ and $\mathrm{BCC} / \mathrm{HCP}$ structured $\mathrm{Zr}$ were not observed. Instead, all three patterns revealed a broad amorphous peak ranging from $30-45^{\circ} 2 \theta$, see Fig. 2 .

The amorphous peak was observed to shift to a smaller angle as the $\mathrm{Zr}$ content was increased. The inter-atomic distance that correspond to these $2 \theta$ values are within the range expected of the $\mathrm{V}_{2} \mathrm{Zr}$ Laves phase [28]. This shift of the central peak location with increasing $\mathrm{Zr}$ content is attributed to the significantly larger atomic radius of $\mathrm{Zr}$ $(1.60 \AA)$ relative to $\mathrm{V}(1.34 \AA)$ [29], which will increase the average distance between atoms and hence produce a higher average inter-atomic distance.

TEM of the three thin films was used to confirm the amorphous nature of the three $\mathrm{V}-\mathrm{Zr}$ compositions at the atomic scale. Figure 3 (a) displays a representative selected area diffraction pattern of a V-Zr film, showing diffuse rings characteristic of an amorphous material. The high resolution BF-STEM image in Figure 3 (b) exhibits the small randomly oriented "fringes" expected for an amorphous material. This kind of phase contrast arises from a randomization of the electron phase by the disordered object, subsequently convolved by the point spread function of the microscope [30]. There are no extended lattice fringes indicative of nano crystallites. This amorphous diffraction pattern was observed from room temperature to $773 \mathrm{~K}$.

\subsection{Modelling the Amorphous Structures}

The experimental results suggest that, in the absence of sufficient thermal activation (or time) to nucleate and grow a dual-phase microstructure (expected when considering the $\mathrm{V}$-Zr phase diagram) an amorphous structure forms. It is interesting, therefore, to probe these structures further, in order to determine the amorphous films' characteristics.

Modelling non-symmetric structures is somewhat difficult due to the periodicity imposed by the supercell method (especially in the limited system sizes feasible using DFT methods). Care must be taken to use sufficiently large supercells, reducing the effect of imaging effects imposed by the periodicity, as well as carefully choosing the supercell's starting density. As a result of the random nature of the amorphous structures, a representative sample of supercells are needed for each composition and the combined results are analysed. This should negate the effects of any accumulation and clustering of like elements which has been said to effect an amorphous system's stability [31], furthermore, individual systems with lattice energies that were outside the statistical range of normally distributed data were removed as outliers.

The effect of the initial supercell's density on the geometry optimised structure was initially considered. For each stoichiometry 54 atoms were placed in a cubic supercell with edge lengths of $9 \times 9 \times 9 \AA, 10 \times 10 \times 10 \AA$, and $11 \times 11 \times 11 \AA$, henceforth referred to as $9 \AA, 10 \AA$, and $11 \AA$ respectively. The averaged simulated $\mathrm{X}$-ray diffraction patterns of the optimised supercells show a distinct change from a crystalline structure with defined peaks at high densities, to the broad amorphous peaks with the lower starting density. The peaks observed in the $9 \AA$ and $10 \AA$ supercells are indicative of crystalline ordering, see Figure 4.

When considering the volume and total energy of each supercell, across the three stoichiometries, the $9 \AA$ supercells were both smaller in volume and higher in energy (less favourable) compared to the larger $10 \AA$ and $11 \AA$ systems. When increasing the starting density to $10 \AA$ the supercells minimise in volume during the structure optimisation and yield a lower energy per atom and higher volume per atom than the $9 \AA$, yet still display sharp peaks within their XRD pattern. Finally, the $11 \AA$ supercells, which are relatively similar in volume and energy per atom to that of the 
$10 \AA$, yield quite dissimilar XRD patterns. This suggests that the initial density of the supercell plays a pivotal role in accurately modelling an amorphous structure. Independent of the optimisation process, a more open structure is initially required to prevent intermediate scale crystalline periodicity in the small supercells used in this approach. For $2 \mathrm{~V}: 1 \mathrm{Zr}$, a second set of $9 \AA$ supercells (see Figure 5) was run with a finer energy convergence criteria of $10^{-4} \mathrm{eV}$, which both expanded in volume and reduced in energy but did not have an effect on the perceived degree of crystallinity.

The effect of scaling, by doubling and halving both starting volume and number of atoms in the supercell, was explored. The smaller supercells exhibited sharper peaks analogous to the $9 \AA$ supercells whereas the larger supercells show a similar broad amorphous hump to that of the $11 \AA$ system. The average lattice energy per atom of the larger and smaller supercells varied from the $11 \AA$ by $1.7 \times 10^{-2} \mathrm{eV}$ and $3.2 \times 10^{-2} \mathrm{eV}$, respectively. These results show that there is little further benefit in scaling to a larger supercell and that it is difficult to effectively simulate an amorphous material with a smaller system than those chosen in this study. For these reasons the $11 \AA \mathrm{su}-$ percells were used for comparison to the experimental data and further analysis.

When comparing the simulated XRD patterns of the modelled $2 \mathrm{~V}: 1 \mathrm{Zr}, 1 \mathrm{~V}: 1 \mathrm{Zr}$ and $1 \mathrm{~V}: 2 \mathrm{Zr}$ structures there is also a distinct similarity in the shift in CPL to that seen in the experimental equivalent, see Figure 6 . To account for the slight difference in stoichiometry between model and experiment, a linear function was applied to the CPL (A) with Zr content (at.\%). The difference in gradient and y-intercept was determined to be $1.3 \times 10^{-3} \frac{\AA}{a t . \%}$ and $0.05 \AA$ respectively. The statistical error associated with the simulated central peak location was calculated to be on the order of $10^{-3} \AA$. For this reason it is clear both experimental and simulated results are in strong agreement.

To investigate this shift in bond length further, a RDF was calculated for the modelled data to determine the approximate distance of first and second nearest neighbours in the three amorphous stoichiometries, see Figure 7. Unsurprisingly, the trend in the shift is in agreement with the XRD data. Further, the RDF produced by all three stoichiometries is in agreement with other experimental and theoretical predictions of amorphous solids showing short range order which diminishes with distance [32, 33, 34]. The data from the RDF should only be considered relevant up until the $\mathrm{L} / 2$ value of $5.5 \AA$ (where $\mathrm{L}$ is the shortest edge length of the supercell).

When considering the average inter-atomic distance of the first nearest neighbours with the change in $\mathrm{Zr}$ content for $(\mathrm{V}, \mathrm{Zr})_{\mathrm{am}}, \mathrm{V}_{2} \mathrm{Zr}, \mathrm{BCC} \mathrm{V}, \mathrm{BCC} \mathrm{Zr}$, and $\mathrm{HCP} \mathrm{Zr}$ we find that there is a variance in the behaviour of the systems with regards to Vegard's law [35], see Figure 8. The amorphous systems have longer average bond lengths to their nearest neighbours than the predicted crystalline
V-Zr systems. This suggests the amorphous systems are more openly packed. The predicted densities of the three stoichiometries are $6.28,6.23$ and $6.46 \mathrm{~g} / \mathrm{cm}^{3}$ for $2 \mathrm{~V}: 1 \mathrm{Zr}$, $1 \mathrm{~V}: 1 \mathrm{Zr}$ and $1 \mathrm{~V}: 2 \mathrm{Zr}$, respectively. These densities exist between that of BCC V $\left(6.00 \mathrm{~g} / \mathrm{cm}^{3}\right)$ and HCP $\mathrm{Zr}(6.52$ $\left.\mathrm{g} / \mathrm{cm}^{3}\right)[36]$ but display a non-linear trend. The difference in trend between average bond length and density with varying $\mathrm{Zr}$ content could be related to short range ordering of polyhedra [37] and/or alternations to their packing density. Cheng and Ma [38] describe the short range polyhedral ordering as less frustrated when atoms of different sizes are present and chemically compatible. The review highlights the variation in structure throughout amorphous metals, some areas being more ordered than others, and relates this to connectivity/poly-tetrahedral packing. Further study of the short range ordering within the $\mathrm{V}-\mathrm{Zr}$ amorphous system using experiment and modelling is required.

\subsection{Thermodynamic stability of the phases}

After gaining some confidence in simulating the amorphous structures in the V-Zr system, the stability in comparison to the crystalline phases is now investigated computationally. Each stoichiometry is considered separately, initially focusing on the $2 \mathrm{~V}: 1 \mathrm{Zr}$ stoichiometry that is expected to form the intermetallic $\mathrm{V}_{2} \mathrm{Zr}$ under certain circumstances. This work only considers the enthalpy of formation. Future work should consider effects such as vibrational entropy as these are expected to have an impact on system stability [39]. Calculations of vibrational effects are currently extremely computationally expensive (as they cannot use symmetric effects to their advantage in the manner crystalline systems are able to do [39]).

\subsection{1. $V_{2} Z r$}

Considering the formation of $\mathrm{V}_{2} \mathrm{Zr}$, we calculate that the enthalpy for formation of the intermetallic to be positive (unfavourable), in agreement with literature [12]:

$$
\begin{array}{r}
2 \mathrm{~V}_{\mathrm{BCC}}+\mathrm{Zr}_{\mathrm{BCC}} \rightarrow \mathrm{V}_{2} \mathrm{Zr}_{\text {Laves }} \\
\qquad \Delta \mathrm{H}=+0.12 \mathrm{eV} / \text { atom }
\end{array}
$$

and

$$
2 \mathrm{~V}_{\mathrm{BCC}}+\mathrm{Zr}_{\mathrm{HCP}} \rightarrow \mathrm{V}_{2} \mathrm{Zr}_{\text {Laves }}
$$

$$
\Delta \mathrm{H}=+0.15 \mathrm{eV} / \text { atom }
$$

at low temperatures $\mathrm{V}_{2} \mathrm{Zr}$ is predicted to be unstable relative to its constituent elements, in agreement with the work of Lumley et al. [12] but in disagreement with the accepted equilibrium phase diagram (meaning it may be kinetically stabilised at room temperature). Of course, the metastable solid solution (whether BCC or amorphous) is even more energetically unfavourable relative to a mixture of the elements, for example:

$$
\begin{aligned}
& 2 \mathrm{~V}_{\mathrm{BCC}}+\mathrm{Zr}_{\mathrm{HCP}} \rightarrow(2 \mathrm{~V}: 1 \mathrm{Zr})_{\mathrm{BCC}} \\
& \Delta \mathrm{H}=+0.40 \mathrm{eV} / \text { atom }
\end{aligned}
$$


Nevertheless, nucleation of a dual-phase structure of the elements requires thermal activation and would obviously be very slow at lower temperatures so the possibility of a massive (i.e. no long range mass transfer) transformation between the phases of the same composition but different structures is also worth considering:

$$
\begin{aligned}
&(2 \mathrm{~V}: 1 \mathrm{Zr})_{\mathrm{BCC}} \rightarrow(2 \mathrm{~V}: 1 \mathrm{Zr})_{\mathrm{am}} \\
& \Delta \mathrm{H}=-0.15 \mathrm{eV} / \text { atom }
\end{aligned}
$$

and

$$
\begin{array}{r}
(2 \mathrm{~V}: 1 \mathrm{Zr})_{\mathrm{am}} \rightarrow\left(\mathrm{V}_{2} \mathrm{Zr}\right)_{\text {Laves }} \\
\qquad \Delta \mathrm{H}=-0.11 \mathrm{eV} / \text { atom }
\end{array}
$$

Therefore, on the basis of these calculations, it can be predicted that a physical vapour deposit would very likely form as $(\mathrm{V}-\mathrm{Zr})_{\text {am }}$ (agreeing with the experimental observations), with transformation to $\mathrm{V}_{2} \mathrm{Zr}$ likely if the sample is subsequently heat treated to a sufficiently high temperature (in agreement with Eickert [7]).

\subsection{2. $1 \mathrm{~V}: 1 \mathrm{Zr}$ and $1 \mathrm{~V}: 2 \mathrm{Zr}$}

We now explore the stability of materials with either the $1 \mathrm{~V}: 1 \mathrm{Zr}$ or $1 \mathrm{~V}: 2 \mathrm{Zr}$ stoichiometry, starting with either a BCC or an amorphous structure. The key question is whether it will be energetically favourable for these structures to transform to a dual-phase mixture of $\mathrm{V}_{2} \mathrm{Zr}$ Laves phase $+\mathrm{Zr}$, or not. The relevant transformations are given in Equations (6) to (8)for $1 \mathrm{~V}: 1 \mathrm{Zr}$ :

$$
\begin{aligned}
2(1 \mathrm{~V}: 1 \mathrm{Zr})_{\mathrm{BCC}} \rightarrow \mathrm{V}_{2} \mathrm{Zr}_{\text {Laves }}+\mathrm{Zr}_{\mathrm{HCP}} \\
\Delta \mathrm{H}=-0.42 \mathrm{eV} / \text { atom }
\end{aligned}
$$

$$
\begin{aligned}
2(1 \mathrm{~V}: 1 \mathrm{Zr})_{\mathrm{am}} \rightarrow \mathrm{V}_{2} \mathrm{Zr}_{\text {Laves }}+\mathrm{Zr}_{\mathrm{HCP}} \\
\Delta \mathrm{H}=-0.16 \mathrm{eV} / \text { atom }
\end{aligned}
$$

$$
\begin{aligned}
&(1 \mathrm{~V}: 1 \mathrm{Zr})_{\mathrm{am}} \rightarrow \mathrm{V}_{\mathrm{BCC}}+\mathrm{Zr}_{\mathrm{HCP}} \\
& \Delta \mathrm{H}=-0.28 \mathrm{eV} / \text { atom }
\end{aligned}
$$

The reactions that can take place with the $1 \mathrm{~V}: 2 \mathrm{Zr}$ stoichiometry are provided in the following equations:

$$
\begin{array}{r}
2(1 \mathrm{~V}: 2 \mathrm{Zr})_{\mathrm{BCC}} \rightarrow \mathrm{V}_{2} \mathrm{Zr}_{\text {Laves }}+3 \mathrm{Zr}_{\mathrm{HCP}} \\
\Delta \mathrm{H}=-0.53 \mathrm{eV} / \text { atom }
\end{array}
$$

$$
\begin{aligned}
2(1 \mathrm{~V}: 2 \mathrm{Zr})_{\mathrm{am}} \rightarrow \mathrm{V}_{2} \mathrm{Zr}_{\text {Laves }}+3 \mathrm{Zr}_{\mathrm{HCP}} \\
\Delta \mathrm{H}=-0.15 \mathrm{eV} / \text { atom }
\end{aligned}
$$

$$
\begin{aligned}
&(1 \mathrm{~V}: 2 \mathrm{Zr})_{\mathrm{am}} \rightarrow \mathrm{V}_{\mathrm{BCC}}+2 \mathrm{Zr}_{\mathrm{HCP}} \\
& \Delta \mathrm{H}=-0.22 \mathrm{eV} / \text { atom }
\end{aligned}
$$

It is clear from the above that the BCC solid solution is unstable relative to all the other considered possibilities whilst the most stable structures are always the dual-phase mixtures of the elements which remain energetically favourable relative a dual-phase microstructure of $\mathrm{V}_{2} \mathrm{Zr}$ and $\mathrm{Zr}$. Nevertheless, in the absence of sufficient thermal activation (and time) to nucleate and grow either of the dual-phase micro-structures, it might be expected that the amorphous solid solution would be found and the amorphous structures will have a tendency to decompose to a dual phase microstructure (see equations 5 - 11) with associated negative formation enthalpies.

These enthalpies of formation may be correlated with the drive of the amorphous structure to crystallise and segregate into nanoscale domains of $\mathrm{V}_{2} \mathrm{Zr}$ and $\mathrm{Zr}$, however the kinetic effects required to form such a heterogeneous microstructure are neglected in equations $(1-11)$. Indeed, kinetic effects such as thermal activations at low temperatures have been well noticed and studied in amorphous structures [40]. These thermal activations were found to play a key role in the temperature dependant behaviour and structure of amorphous metal systems. Nevertheless, we have shown in this section that considering enthalpy alone can provide some useful predictions of materials behaviour in amorphous metal systems.

\section{Conclusions}

Crystallisation of the $\mathrm{V}_{2} \mathrm{Zr}$ Laves phase in $\mathrm{V}-\mathrm{Zr}$ alloys is predicted from the phase diagram but requires relatively high temperatures to occur. Instead, mixtures of $\mathrm{V}$ and Zr that are co-deposited at room temperature using magnetron sputtering have been observed to form amorphous solid solutions. Calculations of formation enthalpies confirmed that an amorphous solid solution would be significantly more stable than a random body-centered solid solution of the elements, in agreement with the experimental results.

We demonstrate how DFT may be used to model the amorphous structure of three stoichiometries $2 \mathrm{~V}: 1 \mathrm{Zr}, 1 \mathrm{~V}: 1 \mathrm{Zr}$ and $1 \mathrm{~V}: 2 \mathrm{Zr}$. The effect of varying starting densities of the supercells were investigated. It was found that a larger more open structure is required, prior to the optimisation process to effectively simulate an amorphous system. Smaller supercells tended to crystallise and displayed sharp peaks similar to that of $\mathrm{V}_{2} \mathrm{Zr}$ and $(\mathrm{V}, \mathrm{Zr})_{\mathrm{BCC}}$.

Similar trends in both simulated and experimental XRD patterns, related to the average inter-atomic distance, were identified by the shift in central peak location with varying $\mathrm{Zr}$ content. This was due to the relatively large atomic radius of $\mathrm{Zr}$ to $\mathrm{V}$ causing an increase in average inter-atomic distance. The modelled amorphous systems were further characterised through a radial distribution function. A positive deviation from Vegard's Law was identified in the distance to the first nearest neighbour. Changes to the nearest-neighbour distances in the three formulations studied may indicate changes in the short-range order of 
the amorphous structures that alter crystallisation pathways.

\section{Acknowledgements}

This work was supported by the Multi-modal Australian ScienceS Imaging and Visualisation Environment (MASSIVE) (www.massive.org.au). We would like to thank Joanne Etheridge, Geoff McCredie, Robert Aughterson and Lyndon Edwards for their help and support. ACYL would like to acknowledge the support of the Science Faculty, Monash University and the assistance of Matthew Weyland of the MCEM. The electron microscopy was conducted in part at the Monash Centre for Electron Microscopy. The FEI Titan ${ }^{3}$ 80-300 FEGTEM was funded by the Australian Research Council (Contract No. LE0454166). ACYL, DJK, SCM and GRL would like to acknowledge support from the ANSTO-Monash collaborative fund. SCM would like to thank Lars Hallstadius of Westinghouse Electric Sweden for his conversations on the subject.

\section{Figures}

- Figure 1 - Glass-forming zone in V-Zr system (adapted and redrawn from Eickert et al. [7])

- Figure 2 - Experimental XRD patterns (dashed) for $1 \mathrm{~V}: 2 \mathrm{Zr}$ (red), 1V:1Zr (black), 2V:1Zr (blue) amorphous thin films with the central peak location indicated by vertical solid lines of matching colour.

- Figure 3 - Representative (a) selected area diffraction pattern of the $1 \mathrm{~V}: 1 \mathrm{Zr}$ film and (b) BF-STEM image of the same amorphous film demonstrating its amorphous nature.

- Figure 4 - Average simulated XRD patterns of optimised: A. $1 \mathrm{~V}: 2 \mathrm{Zr}$, B. $1 \mathrm{~V}: 1 \mathrm{Zr}$, and C. $2 \mathrm{~V}: 1 \mathrm{Zr}$ supercells with starting edge lengths of $11 \AA, 10 \AA$, and $9 \AA$ located at the top middle and bottom, respectively. These patterns are compared to D. (1V:1Zr) $)_{\mathrm{BCC}}$ (grey) and $\mathrm{V}_{2} \mathrm{Zr}$ (black) simulated XRD patterns.

- Figure 5 - Lattice energy per atom (normalised to $\mathrm{V}_{2} \mathrm{Zr}$ ) vs volume per atom of amorphous supercells of $1 \mathrm{~V}: 2 \mathrm{Zr}$ (top), 1V:1Zr (middle), 2) 2V:1Zr (bottom) with starting densities $9 \AA$ : (a) converged to $10^{-3} \mathrm{eV}$ (square), (b) converged to $10^{-4} \mathrm{eV}$ (diamond), $10 \AA$ (circle) and $11 \AA$ (triangle) with $\mathrm{V}_{2} \mathrm{Zr}$ as reference (cross).

- Figure 6 - Average simulated XRD patterns (dashed) for $11 \AA$ IV:2Zr (red), 1V:1Zr (black), 2V:1Zr (blue) amorphous supercells with CPL indicated by vertical solid lines of matching colour.
- Figure 7 - Average simulated radial distribution functions (dashed) for $2 \mathrm{~V}: 1 \mathrm{Zr}$ (red), 1V:1Zr (black), 1V:2Zr (blue) amorphous supercells with CPL of $1^{\text {st }}, 2^{\text {nd }}$ and $3^{\text {rd }}$ nearest neighbours indicated by vertical solid lines of matching colour.

- Figure 8 - Calculated average inter-atomic distances to the first nearest neighbour for $1 \mathrm{~V}: 2 \mathrm{Zr}, 1 \mathrm{~V}: 1 \mathrm{Zr}$, and $2 \mathrm{~V}: 1 \mathrm{Zr}$ (crosses), compared to the modelled ideal crystalline structures of BCC V (circle), HCP (diamond) and $\mathrm{BCC}$ (square) $\mathrm{Zr}$ and $\mathrm{V}_{2} \mathrm{Zr}$ (triangle). Two linear functions (red and blue), representing Vegard's law, are fitted between BCC V and HCP Zr, and $\mathrm{BCC} \mathrm{V}$ and $\mathrm{BCC} \mathrm{Zr}$, respectively.

[1] Shirokov, V. Material Science, 1999;3:401.

[2] Sears, V. Neutron News 1992;3:29.

[3] Smith CO. Nuclear Reactor Materials, Michigan: AddisonWesley; 1967.

[4] Ursu, I. Physics and Technology of Nuclear Materials, Bucharest: Pergamon Press; 1985.

[5] Smith, D., Chung, H., Loomis, B., Matsui, H., Votinov, S., and Witzenburg, W. Fusion Eng Des, 1995;29:399.

[6] Herr, U. and Samwer, K. J Non-Cryst Solids, 1993;126:608.

[7] Eickert, S., Hecht, H., and Minnigerode, G. Z Phys B Con Mat, 1992;88:35.

[8] Matolin, V., Masek, K., Matolinova, I., Skala, T., and K., Veltruska. Appl Surf Sci, 2004;235:202.

[9] Chihi, T., Fatmi, M., and Ghebouli, B. American Journal of Modern Physics, 2013;2:88.

[10] Štrof, J., Pavlu, J., Wdowik, U., J., Buršík, Šob, M., and Vřeštál, J. CALPHAD, 2014;44:62.

[11] Zhao, X., Yuan, G., Yao, M., Yue, Q., and Shen, J. CALPHAD, 2012;36:163.

[12] Lumley, S., Murphy, S., Burr, P., Grimes, R., Chard-Tuckey, P., and Wenman, M. J Nuc Mater, 2013;437:122.

[13] Liu, Y., Yu, D., L., Zhang., and Ge, Y. CALPHAD, 2009;33:425.

[14] Young, D.A. Phase Diagrams of the Elements. Virginia: University of California; 1975.

[15] Hume-Rothery, W. Atomic Theory for Students of Metallurgy. London: Institute of Materials for the Metals and Metallurgy Trust of the Institute of Metals and the Institute of Metallurgists; 1969.

[16] Enomoto, M. J Phase Equilib, 1992;13:206.

[17] Kabliman, E., Mirzoev, A., Phys Met Metallogr, 2009;108:461.

[18] Bishop, C., Grimes, R., Parfitt, D., Nucl Instrum Meth B, 2010;268:2915.

[19] Kresse, G. and Hafner, J. Phys Rev B, 1993;47;558.

[20] Kresse, G. and Hafner, J. Phys Rev B, 1994;49:14251.

[21] Zhang, Y., Zuo, T., Cheng, Y., and Liaw, P. Scientific Reports, 2013;3:1.

[22] Rian, F., Delczeg, L., Chen, N., Varga, L., Shen, J., and Vitos, L. Phys Rev B, 2013;88:085128.

[23] Middleburgh, S., King, D., Lumpkin, G., Cortie, M., Edwards, L., J Alloy Compd, 2014;599:179.

[24] Wang, H., Chroneos, A., Jiang, C., and Schwingenschlogl, U. Phys Chem Chem Phys, 2012;14:11737.

[25] Chroneos, A., Jiang, C., Grimes, R., and Schwingenschogl, U. Chem Phys Lett, 2010;493:97.

[26] Lumpkin, G.R., Smith, K.L., Blackford, M.G., Giere, R., Williams, C.T. Micron, 1994;25: 581.

[27] Oganov, R. and Glass, C. J Chem Phys, 2006;124:244704.

[28] Fruchard, D., Rouault, A., Showmaker, C., Shoemaker, D., J Less-Common Met, 1980;73:363.

[29] Mantina, M., Valero, R., Cramer, C., and Truhlar, D. Atomic radii of the elements, In: CRC Handbook of Chemistry and Physics, Internet Version: Taylor and Francis Group LLC; 2013.

[30] Howie, A. Philos Mag. 2010;90:4647.

[31] Fan, Y., Iwashita, T., Egami, T. Phys Rev E, 2014;89: 062313. 
[32] Sheng, H., Luo, W., Alamgir, F., Bai, J., Ma, E. Nature, 2006;439:419.

[33] Stechert, T., Rushton, M., Grimes, R., Dillon, A. Journal of Non-Crystalline Solids, 2012;358;1917.

[34] Štich, I., Car, R., Parrinello, M. Physical Review B, 1991;44:11092.

[35] Vegard, L. Zeitschrift fur Physik, 1921;5:17.

[36] Hammond, C. The Elements, In: CRC Handbook of Chemistry and Physics, Internet Vesion: Taylor and Francis Group LLC; 2014.

[37] Wakeda, M., Shibutani, Y., Ogata, S., Park, J. Intermetallics, 2007; $15: 139$

[38] Cheng, Q. Ma, E. Progress in Materials Science, 2011;56:379

[39] Lumley, S., Grimes, R., Murphy S., Burr, P., Chroneos, A., Chard-Tucke, P., Wenman, M. Acta Mater, 2014;79:351.

[40] Rodney, D., Tanguy, A., Vandembroucq, D. Model Simul Mater Sc, 2011;19:49. 